\title{
Low-frequency parametric systems in the shallow sea
}

\author{
Sergei Tarasov ${ }^{1}$, Zhu Jianjun ${ }^{2}$, Petr Pivnev ${ }^{1 *}$, and Mikhail Gubko ${ }^{3}$ \\ ${ }^{1}$ Southern Federal University, Taganrog, Russia, \\ ${ }^{2}$ Harbin Engineering University, China \\ ${ }^{3}$ Far Eastern Federal University, Vladivostok, Russia,
}

\begin{abstract}
The theoretical model of acoustic wave propagation in the icewater layer is developed, with, taking into account the velocity dispersion. New opportunities for monitoring the marine environment on long routes, including those covered with ice, are discussed when using parametric sonar antennas. The results of research demonstrating single-mode excitation of a waveguide by a parametric array in a wide frequency band are presented. The possibility of compression of a broadband signal during its propagation, as a result of waveguide dispersion, which leads to an increasing of signal intensity, is shown. The results of experimental studies of the propagation characteristics of a wide-band acoustic signal of a parametric hydroacoustic array in a shallow sea are presented.
\end{abstract}

\section{Introduction}

Multiple factors affect the propagation of acoustic waves in shallow seas. The most significant are: the sound velocity profile, the properties and irregularities of the bottom, the state of the surface, waves, and much more. A significant influence on the propagation of acoustic waves in seas, most of the time covered with ice, has the reflection of sound from the ice border. In this regard, there is a need to study the acoustic parameters and local reflecting properties of pack ice under natural conditions.

Reducing the operating frequencies of sonar systems leads to the need to consider the ice cover as a solid layer, the thickness of which is comparable to the length of the sound wave, and the reflection coefficient is clearly frequency-dependent. In addition, the layered inhomogeneity of elastic parameters of ice, in turn, strongly affects the frequency-angular dependence of the sound reflection coefficient from ice.

In natural marine waveguides, the conditions for the propagation of highly directional sound beams deserve the most attention. Parametric acoustic antennas [1-4] (antennas based on nonlinear interaction of acoustic waves) are a promising tool that can emit a highly directional broadband signal. Their use provides new opportunities for acoustic sensing in the ocean, especially in the marine waveguide. And the high directivity and

\footnotetext{
*Corresponding author: pivnev@mail.ru
} 
broadband of the hydroacoustic antenna based on nonlinear effects allows us to use this tool to study the characteristics of a marine waveguide, including one covered with ice [4-5].

Methods of parametric emission of acoustic waves provide the possibility of selective excitation of modes in a waveguide in a wide frequency band (at least two to three octaves). At the same time, the signal emitted by a parametric array can be perfectly coordinated with the layered structure of the marine environment [5-6].

These qualities of parametric arrays seem to be the most important for researches in shallow areas of the World ocean covered with ice [6].

Thus, the radiating parametric array coordinated with the marine waveguide, due to its features, opens the possibility for nonlinear acoustics methods to include the frequency domain of the waveguide in hydroacoustic research practice, in other words, to use frequency tomography methods instead of spatial tomography.

This new quality of parametric systems creates additional opportunities for monitoring marine areas, including those covered with ice, on long routes. Selective excitation of modes in a wide frequency band in waveguides appears to be the most effective tool for research of acoustic signal compression in marine waveguides and creation of highly effective systems for sea sounding in order to detect submerged objects and inhomogeneities..

\section{Phenomena of waveguide dispersion and compression of a broadband signal in a marine waveguide}

A marine waveguide has a frequency dispersion of the acoustic signal propagation speed. The amount of dispersion depends on the depth profile of the sound velocity and the thickness of the waveguide [6]. Frequency dispersion leads either to the destruction of short broadband pulses that propagate over sufficiently long distances, or to the concentration of energy of the acoustic signal in a short time interval, if the frequency modulation of the signal corresponds to the conditions of dispersion in the medium. In this case, we can say that the acoustic signal is focused or compressed in time.

The prospects for using broadband signals for ocean research are that it is possible to develop a new approach to acoustic tomography of marine areas using a procedure for frequency processing of signals propagating along one route, instead of the known procedure for spatial processing of signals propagating along different routes [7].

Usually the underwater waveguide is formed by the upper warm water layer and the cold intermediate one. Therefore, monitoring the thickness of the waveguide can provide very valuable information about the marine waveguide and changes in its parameters. Direct measurements of the vertical distribution of temperature and salinity on a highway extending for hundreds of kilometers of parameters of the cold intermediate layer are timeconsuming and expensive. Constant monitoring of its dynamics on a long route can be carried out using acoustic methods. The problem of determining the parameters of the layer can be solved by single-mode excitation of acoustic signals in a wide frequency band. Measuring the speed of sound propagation along a long route will allow us to study one of the typical acoustic effects of an underwater waveguide - mode dispersion. Mode dispersion in an underwater wave channel means that the modes of the same number have different group propagation rates at different frequencies. Therefore, broadband signals change their shape during propagation and this change can be experimentally recorded. Measurement of the group velocity will determine the average sound velocity profile in the underwater waveguide.

Since the eigenfunctions of the waveguide are changing according withe characteristics of the sound propagation velocity profile in the waveguide, the study of the eigenfunctions 
of the waveguide in a wide frequency band using parametric arrays can restore the sound velocity profile and provide monitoring of the ocean on long routes.

The propagation conditions in a shallow waveguide in our case correspond to normal waveguide dispersion, when the group propagation speed of the signal increases with frequency. Therefore, to achieve the effect of compression of the broadband signal when it propagates in the waveguide, it is necessary to ensure an increase in frequency over the time of signal emission.

The maximum compression of a signal $\tau$ is determined by the effective frequency band of its spectrum $\Delta f, \tau=(\Delta f)^{-1}$. On the other hand, the duration of the emitted pulse $T$, provided it is fully compressed at a distance $L$, will be determined by the frequency dispersion $\partial c / \partial f$ of the propagation speed of the sound wave c:

$$
T=L \frac{\partial c / \partial f}{c^{2}} \Delta f \text {. }
$$

Thus, when the signal is compressed as a result of waveguide dispersion, its intensity may increase by a factor of $T / \tau$ :

$$
T / \tau=L \frac{\partial c / \partial f}{c^{2}} \Delta f^{2}
$$

In other words, the effect of increasing intensity is proportional to the distance that the signal propagates, the amount of waveguide dispersion, and the square of the frequency band of the signal. This increases the ratio between the signal and the noise that accumulates in the recording equipment during the reception of the signal $\tau$.

Note that the group speed of signal propagation in the waveguide is determined by the waveguide parameters. In particular, for a Pekeris waveguide with a constant sound propagation speed independent of depth $c_{0}$, the frequency dependence of the signal propagation speed is determined by the ratio:

$$
c=c_{0}\left[1-\frac{c_{0}^{2} l^{2}}{(2 f H)^{2}}\right]^{1 / 2},
$$

where $H$ - is the vertical scale of the waveguide, $l$ - is the mode number. Where we get the limit estimate of the frequency dispersion of sound velocity in the waveguide $\partial c / \partial f \approx f^{-2}$. When radiating a signal with a constant relative frequency band $\Delta f / f=$ const, it turns out that the relative compression of the signal $T / \tau$ increases with the distance of the signal propagation $L$. Therefore, the most noticeable effect of the relative increase in the intensity of the broadband signal can be obtained with waveguide propagation on long routes.

\section{Experimental studies of waveguide propagation of a broadband acoustic signal}

Experiments on waveguide propagation of a broadband acoustic signal were conducted in the waters of the Amur Bay and the sea of Azov.

As a radiating parametric array, an array with a pump transducer was used. It was made in the form of a mosaic of radiating piezoelectric elements, half of which radiated a highfrequency pump signal at one frequency, and the other half at another, which differed little in magnitude [8-10]. The average radiation frequency (pump frequency) was $140 \mathrm{kHz}$. The difference frequency or frequency of the signal emission lay in the range of $5 \mathrm{kHz}-50 \mathrm{kHz}$. The electrical power of the array amplifier was $2 \mathrm{~kW}$ for each of the pumping frequencies. The width of the directional characteristic at the level of 0.7 in pressure over the entire operating frequency range was $3 \mathrm{deg}$. 
Figure 1 shows the array beem patterns experimentally measured in a hydroacoustic pool in the range of difference frequencies from 5 to $50 \mathrm{kHz}$. Experimental measurements have shown that beem patterns width remains constant over the entire specified frequency range. This makes it possible to compare the measurement data obtained at all frequencies.

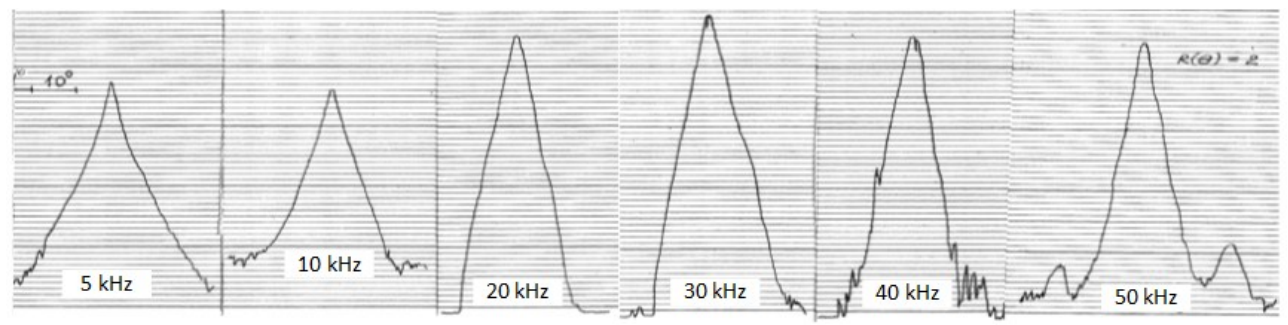

Fig.2. The array beam patterns at different frequencies: 5, 10, 20, 30, 40, and $50 \mathrm{kHz}$.

The radiating array was mounted on a rotary device and could provide scanning of the water area with a narrow beam of parametric radiation in the range of angles $-90^{\circ}-+90^{\circ}$ in the horizontal plane. The array was located at a depth of 1.5-1.7 m and the radiation axis was oriented horizontally. The depth of the place in the experimental area was $2.5-3 \mathrm{~m}$. The receiving array was made in the form of a vertical chain of eight hydrophones, which were located with a step of $0.25 \mathrm{~m}$ on a metal rod. This rod as part of a rigid structure was installed on the bottom in such a way that the vertical chain of hydrophones overlapped the entire waveguide. The signal from the receiving elements of the array was transmitted via cable to the receiving vessel, where it was digitized and recorded for further processing.

The vertical distribution of the speed of sound propagation in the waveguide was determined by the solar heating of its upper part in the daytime. Signals were recorded in parallel from each receiver of the vertical receiving antenna. The measurements were performed at distances between the emitter and the receiving antenna from $1 \mathrm{~km}$ to 5.60 $\mathrm{km}$. The time-frequency characteristics of pulse propagation with a duration of 2 and $4 \mathrm{MS}$ with filling were studied, the frequency of which had linear modulation in the $7 \mathrm{kHz}-20$ $\mathrm{kHz}$ band, while the signal frequency was scanned from the lower frequencies to the upper ones, which corresponded to normal waveguide dispersion.

For Figure 2 an example of recording signals recorded on a vertical chain of hydrophones at a distance about $1000 \mathrm{~m}$ from the emitter is given. It can be seen, that the main radiation energy is concentrated in the middle part of the waveguide. Detailed analysis showed that the signals registered by different hydrophones of the receiving array are in phase along the entire depth of the waveguide, which indicates a predominantly single-mode signal propagation mode. That is, under experimental conditions, the parametric array excited the first mode of the waveguide. Detailed analysis showed that the signals registered by different hydrophones of the receiving array are in phase along the entire depth of the waveguide, which indicates a predominantly single-mode signal propagation mode. In other words, the first waveguide mode was excited under experimental conditions.

Figure 3 shows the vertical distribution of the acoustic field in the waveguide at different distances. The field practically does not oscillate in the vertical plane, which also indicates that the signal propagation can be characterized, as single-mode with a pronounced contribution of the first mode.

Since the waveguide in our case is uniform and the velocity of sound propagation in it is practically independent of depth, the distribution in our case correspond to the normal 
waveguide dispersion, when the group velocity of propagation increases with frequency. Therefore, to achieve the effect of compression of a broadband signal during its propagation in the waveguide, it is necessary to ensure an increase in frequency during the signal emission. This modulation mode was used in our experiments. The calculation of the velocity dispersion makes it possible to estimate the change in the delay of various frequency components of the signal during its propagation in the waveguide.

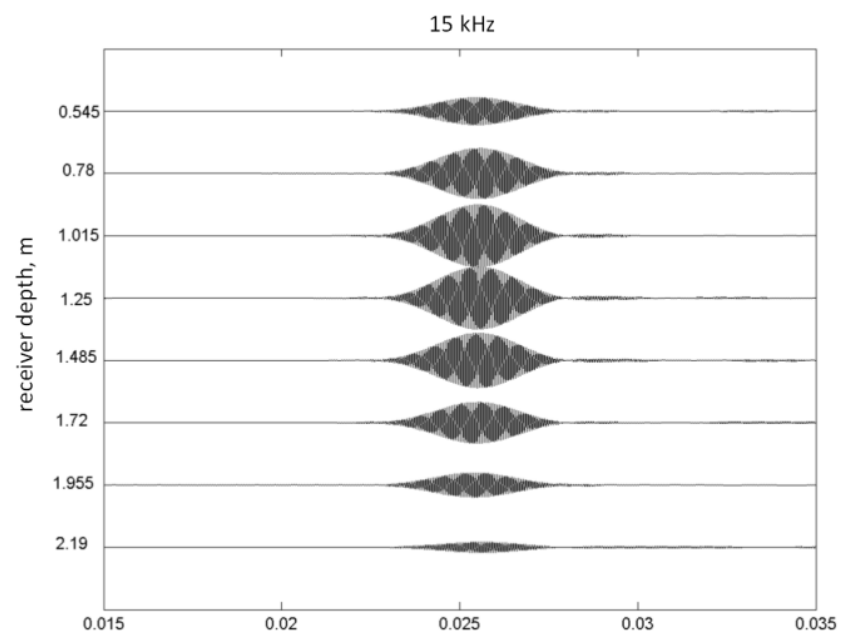

Fig.2. Signals on a hydrophone chain at distance of $1 \mathrm{~km}$. The frequency of $15 \mathrm{kHz}$, pulse length $2 \mathrm{~ms}$.

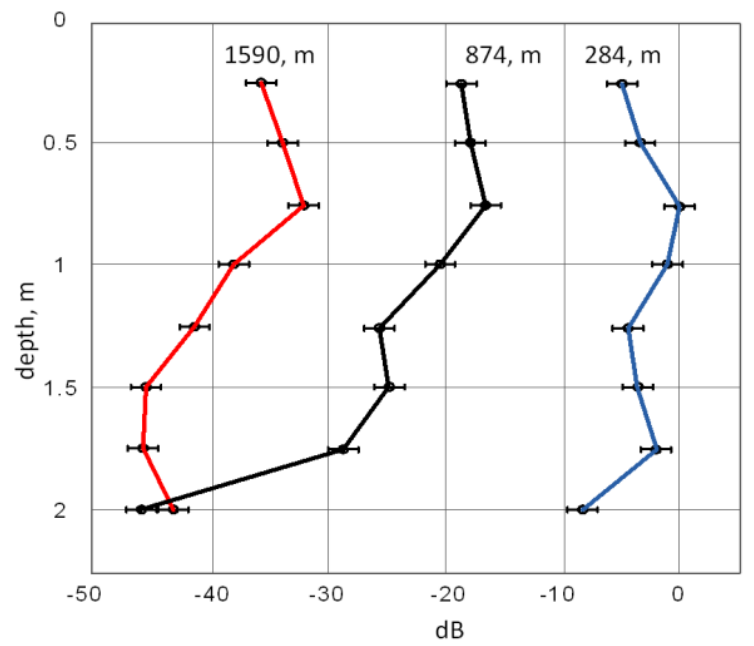

Fig.3. Vertical distribution of the signal over the waveguide cross-section. Hydrophones were located $0.25 \mathrm{~m}$ apart.

The eigenfunction of the first mode is calculated for the measured profile of the sound velocity and the bottom in the form of a liquid half-space with the following parameters: 
the density of the soil at the bottom is $1800 \mathrm{~kg} / \mathrm{m} 3$, and the speed of sound propagation in the ground was considered equal to $1520 \mathrm{~m} / \mathrm{s}$. The speed of sound in water was assumed to be $1499 \mathrm{~m} / \mathrm{s}$. These parameters are typical for the sandy bottom of the sea of Azov. The frequency dependence of the signal propagation velocity in an inhomogeneous shallowwater waveguide was calculated taking into account the conditions at the waveguide boundaries. The upper boundary of the waveguide is free, so the sound pressure on it vanishes. The nature of the distribution and the fact that it is practically independent of the distance also indicates that the signal propagation can be characterized as single-mode.

The calculation of the group velocity dispersion allows us to estimate the change in the delay of various frequency components of the signal during its propagation in a shallow waveguide. Figure 4 shows the dependencies of this delay for different distances traveled by the signal.

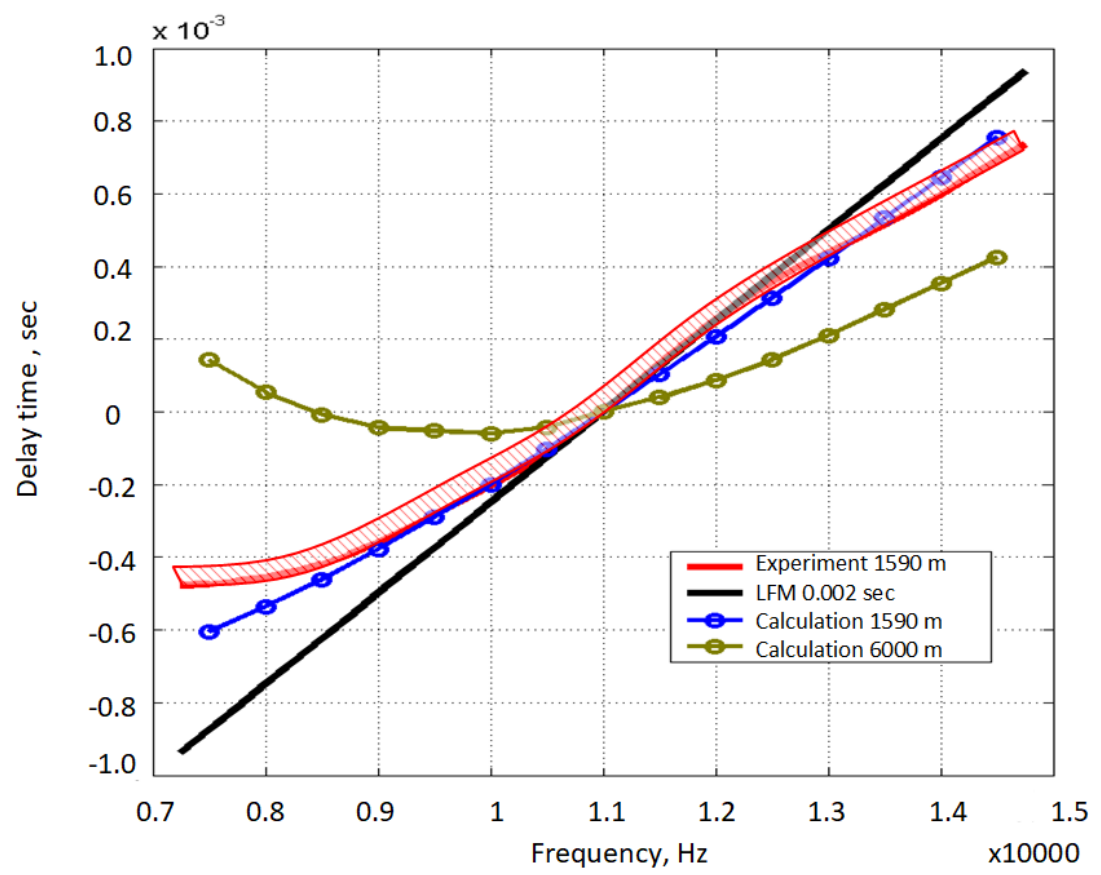

Fig. 4. Changing the delay time of arrival of frequency signals signal components. Results of the experiment and calculation.

As can be seen, the slope of the dispersion curve for the LFM signal practically corresponds to the time-frequency processing of the signal measured at a distance $1590 \mathrm{~m}$. As the distance increases, the time-frequency relationships in the signal are changing. The delay of the low-frequency components of the signal increases. This corresponds to a shorter duration. The analysis shows that the arrival time of low-frequency and highfrequency components can coincide at a distance approximately $6 \mathrm{~km}$ for the conditions of this experiment. Since the dispersion of the signal propagation speed depends on the frequency in a non-linear way, the nature of the frequency modulation must also be nonlinear, in order to obtain the maximum compression of the signal.

The LFM signal used in the experiments did not fully correspond to the character of the waveguide dispersion of the sound velocity detected experimentally. Therefore, compensation for the propagation time of various frequency components of the signal was 
possible only in a limited frequency range. This provided a signal compression of more than 4 times at a distance about $3.8 \mathrm{~km}$. To achieve complete synchronism of the arrival of all frequency components, a special frequency modulation is necessary, corresponding to the features of the dispersion of the sound speed in the waveguide.

Thus, the parametric array, due to its ability to selectively excite waveguide modes in a wide frequency band, is the most effective tool for studying the compression of acoustic signals in ocean waveguides and monitoring the ocean along long routes [8-12]. The results of the experiment coincided quite well with the theoretical calculations, which indicates the prospects for applying the resulting calculation model in similar systems.

\section{Conclusion}

Experimental studies have shown that the dispersion in the waveguide leads to changes in the signal structure, which can be used in spatial signal processing to compress them and thereby increase the range of hydroacoustic systems when working in shallow seas, including those covered with ice. Such unique results obtained using a parametric array were achieved due to their high directivity and constancy of the width of the directivity characteristic in a wide range of difference frequencies [2].

\section{References}

1. V.A. Voronin, S.P. Tarasov, V.I. Tymoshenko, Hydroacoustic parametric systems (Rostizdat, Rostov-on-Don, 2004)

2. P.P. Pivnev, V.A.Voronin, S.P. Tarasov, G.V. Soldatov, Exploration and monitoring of the underwater environment of the shelf zone, ch 9 (2018)

3. B.K. Novikov, O.V. Rudenko, V.I. Timoshenko, Nonlinear hydroacoustics (Sudostroenie, Leningrad, 1981)

4. I.B. Esipov, S.P. Tarasov, V.A. Voronin, O.E. Popov, Nonlinear acoustics fundamentals and applications 18th international Symposium on nonlinear acoustics. Stockholm, Sweden (2008)

5. I.B. Esipov, O.E. Popov, V.A. Voronin, S.P. Tarasov, Acoustic Journal, 1 (55), (2009)

6. Charnotsky M. I., Fuks I. M., Naugolnykh K. A., Smirnov A.V., Di Iorio D., Esipov I. B. Acoustic journal, 2 (52) - 2006.

7. L.M. Brekhovskikh, Yu.L. Lysanov, Theoretical bases of ocean acoustics (Gidrometeoizdat, Leningrad, 1982)

8. I.B. Esipov, O.N. Johannessen, K.A. Naugol'nyck, Yu.Yu. Wang, I.S. Shang, Acoustic Journal, 4 (45), (1999)

9. Yu.S. Kobyakov, N.N. Kudryavtsev, V.I. Tymoshenko, Construction of hydroacoustic fish-searching equipment (Sudostroenie, Leningrad, 1986)

10. V.I. Kaevitser, V.M. Razmanov, A.P. Krivtsov, I.V. Smoljaninov, S.A. Dolotov, Radioengineering, 8, (2008)

11. Mosolov S. S., Sknariya A.V., Tutynin E. V., Zalogin N. N. IV all-Russian conference "radar and radio communication", Moscow, Russia (2010)

12. V.A. Voronin, P.P. Pivnev, S.P. Tarasov, Electronic Scientific Journal "Engineering Journal of Don", 4, (2015) 